\title{
Performance analysis of MIMO communication system with NLOS UV channel
}

\author{
Gleb S. Vasilyev, ${ }^{* 1}$ Oleg R. Kuzichkin, ${ }^{2}$ Dmitry I. Surzhik ${ }^{3}$ \\ ${ }^{1}$ Belgorod State University, Belgorod, 308015, Russia \\ ${ }^{2}$ Belgorod State University, Belgorod, 308015, Russia \\ ${ }^{3}$ Vladimir State University, Vladimir, 600000, Russia
}

Received March 23, 2020; accepted November 19, 2020; published December 17, 2020

\begin{abstract}
Performance analysis is carried out, of a multiple input, multiple output (MIMO) ultraviolet (UV) communication system with a non-line-of-sight (NLOS) UV channel. The achievable bit error coefficient is calculated using three spatial multiplexing methods for different bitrate values, azimuthal deviation between the directiona diagrams of an optical transmitter and an optical receiver, and different noise levels.
\end{abstract}

Communication systems of the solar-blind UV-C range from 200 to $280 \mathrm{~nm}$ enable communication in the absence of direct visibility (non line-of-sight, NLOS) due to strong scattering of UV radiation in the atmosphere, including the presence of high obstacles between the transmitter and receiver, when traditional radio communication is ineffective [1-4]. One of the ways to improve the performance of UV-C communication systems is to use multiple input, multiple output (MIMO) technology [5-6]. In addition, the use of an array of transmitters and an array of receivers oriented in different directions allows for communication when moving and turning communication nodes, which is typical of a mobile ad-hoc network with a UV-C channel [7-9]. To use MIMO technology in real UV communication systems, it is necessary to analyze the performance characteristics of the system in different operating conditions, determined by the channel state and the selected transmission mode.

Calculated energy characteristics of the MIMO communication system with an abstract channel for three spatial multiplexing methods with $N=2$ transmitter channels and $M=2$ receiver channels are shown in Fig. 1. Detection by a receiver is based on one of three criteria: i) maximum-likelihood (ML); ii) suppression of nonlinear interference based on minimizing the average power of interference at the reception (Zero-Forcing Successful Interference Cancellation, ZF-SIC); iii) suppression of nonlinear interference based on minimization of the meansquare-error estimation (Minimum-Mean-Square-Error SIC, MMSE-SIC). To analyze the communication system with a real UV-C channel, it is necessary to determine the

\footnotetext{
*E-mail: vasilievgleb@yandex.ru
}

dependence of the signal-to-noise ratio (SNR) on the channel parameters.

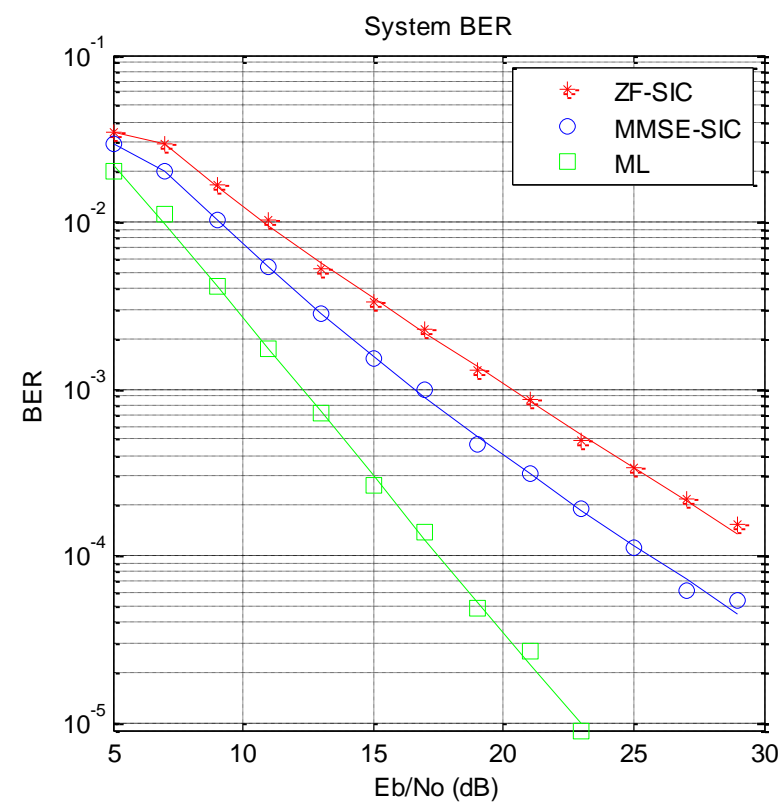

Figure 1. Energy characteristics of the MIMO system for three spatial multiplexing methods.

The signal-to-noise ratio (SNR) of the UV channel is defined as the ratio of the number of detected signal photons to the number of noise photons. The number of signal photons depends on the power and bit rate of the transmission, the losses in the communication channel, the aperture, and the quantum efficiency of the receiver. Typical frequencies for detecting noise photons at low, medium, and high noise levels are about 1000, 5000, and $15,000 \mathrm{~Hz}$, respectively (when using a high-quality solarblind filter and a photoelectronic multiplier with an aperture of $1.92 \mathrm{~cm}^{2}$ ) [10].

The calculated energy characteristics of the MIMO UV communication system are shown in Figs. 2-4. Modeling of the amount of losses in the UV channel is performed on the basis of the Monte Carlo method. The following values of the UV channel parameters were accepted: the communication range $\mathrm{r}=100 \mathrm{~m}$, the elevation angles of 
the transmitter and receiver $\theta_{1}=30^{\circ}$ and $\theta_{2}=30^{\circ}$, the width of the radiation patterns of the transmitter and receiver $\varphi_{1}$ $=10^{\circ}$ and $\varphi_{2}=30^{\circ}$, the radiation wavelength $\lambda=260 \mathrm{~nm}$, the scattering and absorption coefficients for clear weather, the receiver aperture area $\mathrm{A}_{\mathrm{r}}=1.92 \mathrm{~cm}^{2}$ ). Also accepted are $P_{\mathrm{T}}=50 \mathrm{~mW}, S N R=10 \mathrm{~dB}$, and $R=100 \mathrm{kbit} / \mathrm{s}$.

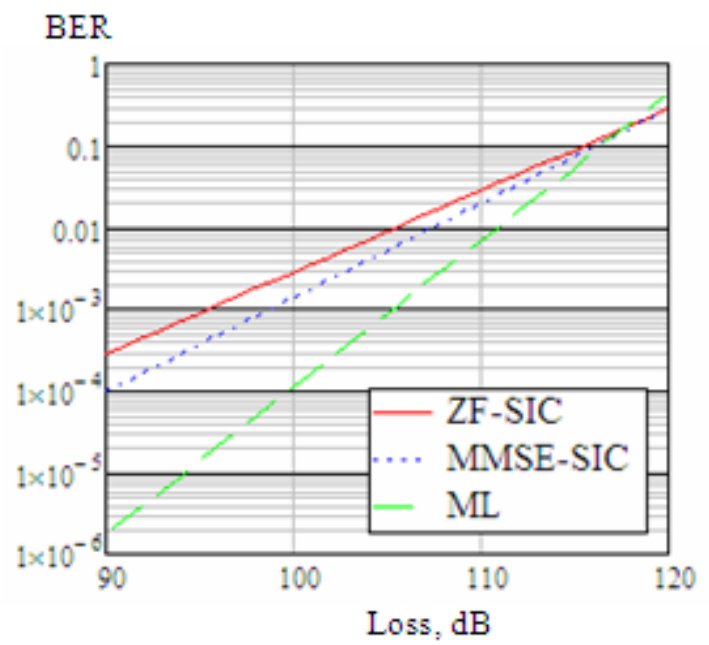

Fig. 2. Dependencies of BER on loss for different types of spatial multiplexing at high noise levels.

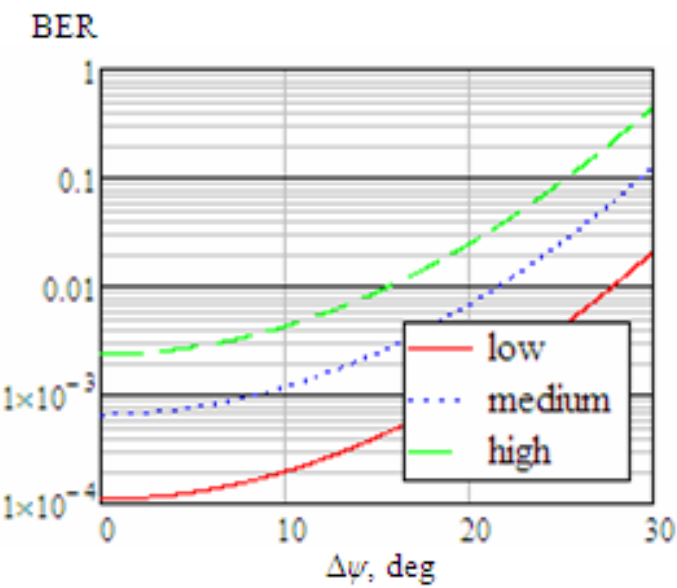

Fig. 3 - Dependences of BER on azimuthal deviation $\Delta \psi$ for MMSESIC at different noise levels.

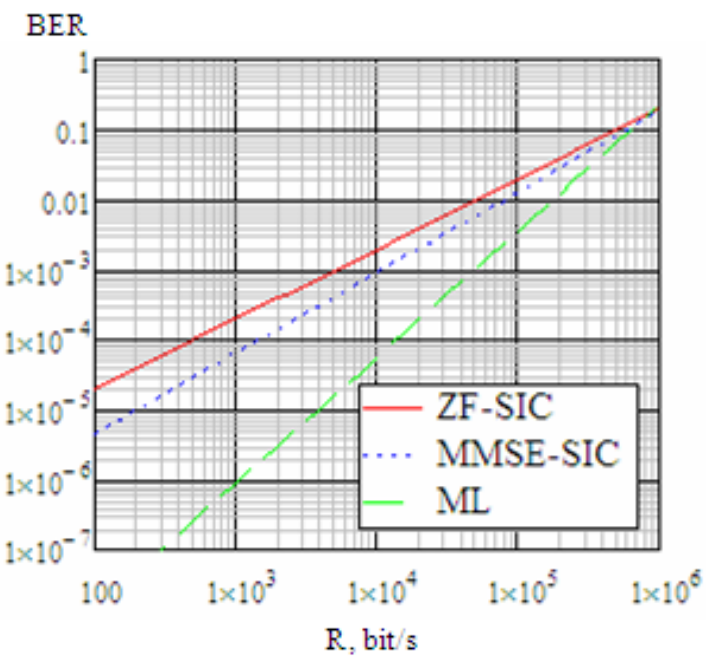

Fig. 4. Dependencies of BER on bitrate for different types of spatial encoding at high noise level.

The multiplexing method based on the maximum likelihood criterion (ML) provides the lowest bit error rate (BER), all other things being equal, but has the highest computational cost. Therefore, in real systems, it may be appropriate to use the MMSE-SIC method with better characteristics than ZF-SIC, but with lower computational costs than ML. The strong dependence of BER on the azimuthal deviation between the directional diagrams of the optical transmitter and the optical receiver $\Delta \psi$ (Fig. 3) determines the need for a high-quality guidance system. The conducted research allows us to determine the performance characteristics of the MIMO UV communication system in various operating conditions (when changing the channel state, transmission mode, turns or movements of communication nodes).

The work was supported by RFBR grant 19-29-06030MK "Research and development of wireless ad-hoc network technology between UAVs and smart city dispatch centers based on adaptation of transmission mode parameters at different levels of network interaction". The theory was prepared in the framework of the state task FZWG -2020-0017 "Development of theoretical foundations for building information and analytical support for telecommunication systems for geoecological monitoring of natural resources in agriculture".

\section{References}

[1] Z. Xu, B. Sadler, IEEE Commun. Mag. 4667-73 (2009).

[2] D. Han, Y. Liu, K. Zhang et al., Opt. Expr. 20(14), 15833 (2012).

[3] Q. Guo, N. He, Z. He, Study Opt. Comm. 3, 64 (2013). 
[4] G. Chen, L. Liao, Z. Li et al., Communication Systems, Networks and Digital Signal Processing (CSND-SP), 9th Int. Symp. on IEEE, 904-909 (2014).

[5] M.A. El-Shimy, S. Hranilovic, J. Lightwave Techn. 33(11), 2246 (2015).

[6] G. Shaw, M. Nischan, M. Iyengar, S. Kaushik, M. Griffin, Proc. SPIE 412683, 96 (2000).

[7] I.S. Konstantinov, G.S. Vasyliev, O.R. Kuzichkin, D.I. Surzhik, I.A. Kurilov, S.A. Lazarev, J. Eng. Adv. Techn. 8(5S) July 2019, DOI:10.35940/ijeat.E1063.0785S319.

[8] I.S. Konstantinov, G.S. Vasilyev, O.R. Kuzichkin, I.A. Kurilov, S.A. Lazarev, J. Adv. Res. Dynam. Contr. Syst. 07, 1853 (2018). http://jardcs.org/abstract.php?archiveid=5147.

[9] I.S. Konstantinov, G.S. Vasyliev, O.R. Kuzichkin, D.I. Surzhik, I.A. Kurilov, S.A. Lazarev, J. Adv. Res. Dynam. Contr. Syst. 11(08), 1920 (2019). http://www.jardcs.org/abstract.php?id=2543

[10] G. Chen, F. Abou-Galala, Z. Xu, B.M. Sadler, Opt. Expr. 16(19), 15059 (2008). 